\title{
ING: INDOOR ROUTING PROTOCOL For GREEN HOME NETWORKS
}

\author{
Kaouthar Sethom ${ }^{1}$, Nozha Al- Ibrahimi ${ }^{2}$ and Guy Pujolle ${ }^{2}$ \\ ${ }^{1}$ Systel Lab, SUPCOM, University of Carthage, Tunisia \\ Firstname.lastnamedesti.rnu.tn \\ ${ }^{2}$ LIP6, University of Paris 6, France \\ Firstname.lastname@lip6.fr
}

\begin{abstract}
Because of all the innovations in computer communications and digital consumer device technologies, people have had great interest in researching home networking systems. The home is evolving rapidly into a smart Mesh networked environment.

Moreover, the energy consumption of wireless devices and networks gradually increases to represent a significant portion of the operational cost, naturally also causing increased equivalent carbon emissions to the environment. Therefore, reducing the energy consumption by several orders of magnitude compared to the current level has become an important requirement for the design of future green wireless networks.

In this paper, we propose an indoor traffic routing algorithm inside green home networks.
\end{abstract}

\section{KEYWORDS}

Autonomic networking, routing, Green communication

\section{INTRODUCTION}

In recent years, the home environment has seen a rapid increase in usage of network enabled digital technologies that offer new and exciting opportunities to increase the connectivity of devices within the home environment for the purpose of home automation. An example of home networks is given in Figure 1.

Wireless Mesh Networks (WMNs) are an emerging technology that could revolutionize the way wireless network access is provided. The interconnection of access points using wireless links exhibits great potential in addressing the last mile connectivity issue in home scenarios. To realize this vision, it is imperative to provide efficient resource management. Resource management encompasses a number of different issues, including routing. Although a profusion of routing mechanisms has been proposed for other wireless networks, the unique characteristics of home mesh networks (e.g., wireless backbone) suggest that it demand a specific solution.

In this paper, we assume general heterogeneous network architecture as shown in Figure 1. There are two basic entities in the system: mobile nodes (MNs) and access points (APs). We will only focus on AP discovery and path selection in the multihop part of the Mesh architecture. 
In contrast with wired networks, energy consumption may represent an essential constraint in wireless mesh home networks. Sensors as well as small and battery-operated wireless devices have restricted battery lifetime and are most vulnerable to the energy constraints. Energy-related objectives are often at odds with performance related objectives. For example, choosing paths so that the overall de-lay(throughput) is minimized may result in overuse of certain nodes in the net-work and premature exhaustion of their battery. Therefore, energy concerns have to be properly reflected in the definition of routing metrics.

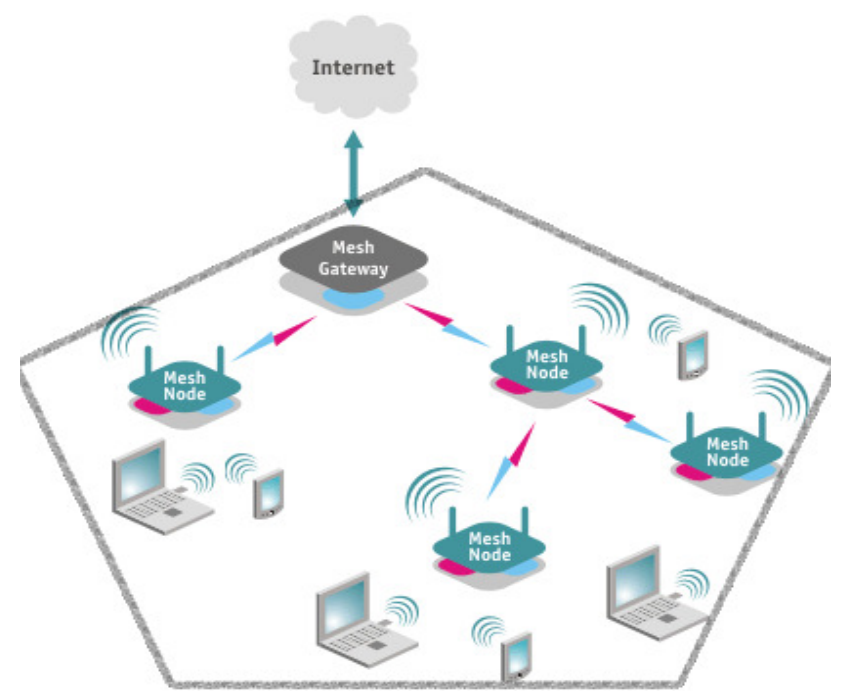

Figure $1:$ Mesh home network topology

The biggest challenge in home network is the need of better connectivity. A set of Adhoc and Mesh routing protocols have been proposed to ensure the network connectivity. From [1], it can be seen that in MANETs [2] [3], the most favored research approach is proactive routing; in sensor networks (WSN) [4] both proactive and reactive approaches are equally used; and in mesh networks, routing approaches are mainly reactive or hybrid (Table 1 ). The choice of a routing technique is made based on the network characteristics with the greatest impact on routing.

Building such routing algorithms in mesh home networks poses a significant technical challenge, since the devices are battery operated. The devices need to be energy conserving so that battery life is maximized. The shortest path is the most common criteria adopted by the conventional routing protocols proposed. The problem is that nodes along shortest paths may be used more often and exhaust their batteries faster. The consequence is that the network may become disconnected leaving disparity in the energy, and eventually disconnected subnetworks. Therefore, the shortest path is not the most suitable metric to be adopted by a routing decision. Other metrics that take the power constraint into consideration for choosing the appropriate route are more useful in the home network.

In this paper, we propose an energy efficient routing algorithms "ING". It is based on a hybrid routing scheme: a proactive dynamic zone is calculated for load balancing through fuzzy logic scheme. The rest of the home devices are organised through an energy-aware reactive routing scheme, since it less expensive in terms of energy consumption than proactive scheme. 
International Journal of Computer Networks \& Communications (IJCNC) Vol.3, No.4, July 2011

Table 1: Qualitative Comparison between adhoc, sensors and mesh routing algorithms

\begin{tabular}{|c|c|c|c|c|c|c|c|c|}
\hline & $\begin{array}{l}\text { Routing } \\
\text { protocols }\end{array}$ & Proactive & $\begin{array}{l}\text { On- } \\
\text { demand }\end{array}$ & Flat & Hierarchical & $\begin{array}{l}\text { Location- } \\
\text { aware }\end{array}$ & Metrics & Mobility \\
\hline \multirow{15}{*}{ Ad hoc } & DSDV & $x$ & & $x$ & & No & Hops & Yes \\
\hline & DSR & & $x$ & $\mathrm{x}$ & & No & Hops & Yes \\
\hline & TORA & & $\mathrm{x}$ & $\mathrm{x}$ & & No & Hops & Yes \\
\hline & CGSR & $\mathrm{x}$ & & & $\mathrm{X}$ & No & vio $\mathrm{CH}$ & Yes \\
\hline & GeoCast & $\mathrm{X}$ & & & $\mathrm{X}$ & Yes & Hops & Yes \\
\hline & $\mathrm{ZRP}$ & $x$ & $x$ & & $x$ & No & $\begin{array}{l}\text { Hops } \\
\text { (zone) }\end{array}$ & Yes \\
\hline & DREAMI & $\mathrm{X}$ & & $\mathrm{X}$ & & Yes & Hops & Yes \\
\hline & LAR & & $\mathrm{X}$ & $\mathrm{X}$ & & Yes & Hops & Yes \\
\hline & OLSR & $\mathrm{x}$ & & $x$ & & No & Hops & Yes \\
\hline & AODV & & $\mathrm{x}$ & $\mathrm{x}$ & & No & Hops & Yes \\
\hline & HSR & $x$ & & & $x$ & No & vio $\mathrm{CH}$ & Yes \\
\hline & FSR & $\mathrm{x}$ & & $\mathrm{x}$ & & No & Hops & Yes \\
\hline & TBRPF & $\mathrm{X}$ & & $\mathrm{X}$ & & No & Hops & Yes \\
\hline & LANMLAR & $X$ & & & $X$ & No & $\begin{array}{l}\mathrm{Hops} \\
\text { (zone) }\end{array}$ & Group \\
\hline & GPSR & $\mathrm{X}$ & & $\mathrm{X}$ & & Yes & Distance & Yes \\
\hline \multirow[t]{8}{*}{ WSN } & LEACH & $x$ & & & $\mathrm{x}$ & No & Energy & Yes \\
\hline & PEGASIS & & $\mathrm{x}$ & & $\mathrm{x}$ & No & Energy & Yes \\
\hline & TEEN & & $x$ & & $\mathrm{X}$ & No & Energy & Yes \\
\hline & SPIN & $\mathrm{X}$ & & $X$ & & No & Energy & No \\
\hline & $\begin{array}{l}\text { Directed } \\
\text { Diffusion }\end{array}$ & & $x$ & $X$ & & No & Energy & Yes \\
\hline & TTDD & $\mathrm{x}$ & & & $\mathrm{x}$ & Yes & Energyr & No \\
\hline & $\begin{array}{l}\text { Rarldom } \\
\text { Walk }\end{array}$ & & $X$ & $\mathrm{X}$ & & No & Energy & No \\
\hline & $\begin{array}{l}\text { Rumor } \\
\text { Routing }\end{array}$ & $\mathrm{X}$ & $\mathrm{x}$ & $\mathrm{X}$ & & No & Energy & Limited \\
\hline \multirow[t]{2}{*}{ WMIN } & MSSR & $x$ & $\mathrm{x}$ & $\mathrm{x}$ & & No & Proprietary & Yes \\
\hline & $5 \mathrm{r} \in \mathrm{RR}$ & & $X$ & $X$ & & No & ETT & $\begin{array}{l}\text { Not } \\
\text { considered }\end{array}$ \\
\hline
\end{tabular}

\section{HYBRID PROTOCOL DESIGN}

In our approach, the ING protocol (Indoor routing protocol for Green Home networks) combines both proactive and reactive mechanisms. The network is divided into two regions:

- Proactive Region: APs and MNs within an m hop radius of an AP are in the proactive region. All MNs maintain active information about AP in this region through periodic Hello packets sent by AP. Hereafter, we call $\mathrm{m}$ as proactive radius. This range determines the AP's discovery scope, called a proactive area. Nodes that are further away have to solicit advertisements reactively instead.

- Reactive Region: All MNs more than $m$ hops away from an AP are part of the reactive region, and use a reactive routing protocol to discover routes to an AP.

Protocol functionality of our proposed protocol can be divided into the following different phases:

1) Hello Message Transmission: All APs periodically transmit Hello packets (denoted by HELLO), which only traverse $\mathrm{m}$ hops (i.e., inside the proactive region), as defined by 
using the TTL value in the IP header. Upon receiving a Hello packet, the node first determines whether it is within m-hop distance from the AP. If so, the route to the AP is created or updated. Only nodes $m_{i} 1$ hops away from the AP decrease the TTL value and rebroadcast the packet.

2) Route Discovery (Proactive Region): A node determines that it is in the proactive region if it has received a Hello packet from any AP. Then, it can start sending data using the information in the routing table without performing route discovery phase.

3) Route Discovery (Reactive Region): RREQ and RREP packets are similar to AODV specifications, but have additional fields to include energy metric. If a node does not have any valid route available to any destination in its routing table, it broadcasts a RREQ.

4) Route Selection: Route selection is related to the cost metric used in the protocol, i.e., energy consumption. The ING protocol selects the route with the best energy saving value for the available destinations. If two or more routes have the same energy value, then the route with maximum life time is selected to forward the packets.

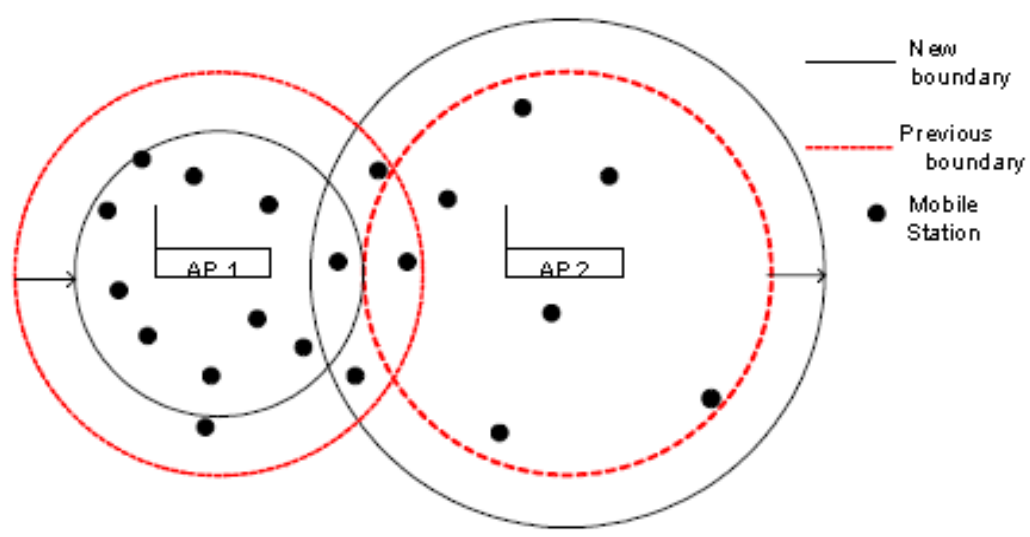

Figure-2 : Proactive AP zone adaptation

The primary challenge in the design of a hybrid approach is how to determine the optimal proactive area. Depended on optimized parameters for given home network, hybrid approach can provide good connectivity while keeping the overhead costs low The loss rate and delay are decreased by increasing the area, but it will pay more in packet overhead to maintain routes in a larger area. The routing overhead is reduced by decreasing the area, but it may pay more in delay and experience higher loss rates [5]. Thus, fixed value of proactive area is not the best choice for all levels of network conditions. To achieve optimal performance, we propose an adaptative AP discovery scheme which dynamically resizes the range of proactive zones.

We use fuzzy logic to finds the optimal area size for each access router with low overheads according to nodes density, route request rate and buffer capacity rate of the AP.

\section{ING ALGORITHM SPECIFICATIONS}

\subsection{A fuzzy logic for radius optimization through power constraint}

The fuzzy can be used to model any continuous function or system. Figure 3 shows the generalized block diagram of fuzzy system. The advantages of fuzzy logic are: 
- conceptually easy to understand

- flexible

- tolerant of imprecise data

- can model nonlinear functions of arbitrary complexity

- can be built on top of the experience of experts

- can be blended with conventional control techniques

- based on natural language

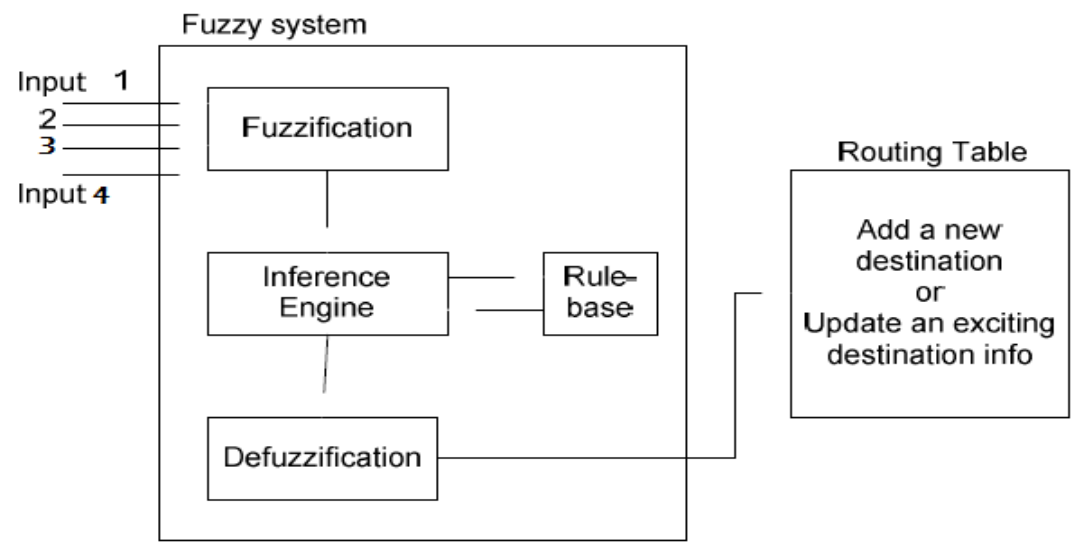

Figure -3: Fuzzy logic system

Fuzzy systems theory or "fuzzy logic" [6] is a linguistic theory that models how we reason with vague rules of thumb and commonsense. The basic unit of fuzzy function approximation is "ifthen" rules. A fuzzy system is a set of if- then rules that maps input to output. The Fuzzy system used here is a Mamdani type [7] system with four inputs (Buffer occupancy rate, route request rate and Nodes density, Energy constraint) and one output (zone radius).

Fuzzy logic has been applied to problems that are either difficult to tackle mathematically or where the use of fuzzy logic provides improved performance. In this paper, fuzzy logic is used to resolve a multi-parameters radius optimization problem. Each Access point needs to optimize its proactive radius depending on its buffer occupancy rate, route request rate and Nodes density in vicinity. An energy constraint is imposed to reduce AP power consumption.

These four decision parameters reflect the network status and nodes conditions. These parameters are updated periodically evry $t$ minutes. The fuzzifier converts the three crisp inputs into suitable linguistic values which are needed by the inference engine.

According to the home domain knowledge, we set three input linguistic variables as: $\mathrm{C}(\mathrm{Buffer}$ occupancy rate $)=\{$ High, Medium, Low $\}=\{\mathrm{HI}, \mathrm{ME}, \mathrm{LO}\} ; \mathrm{C}$ (route request rate $)=\{$ High, Medium, Low $\}=\{$ HI, ME, LO $\}$; C(Nodes density $)=\{$ More, Medium, Few $\}=\{$ MO, ME, FE $\}$. Figure 4 illustrates the membership functions of the input variables.

The Fuzzy rules have IF-Then structure. The inputs are then combined using the And operator. The following is an example of rules which describe the input output mapping:

IF buffer occupancy is Empty AND the node density is Large AND route request Medium then the zone radius is High 

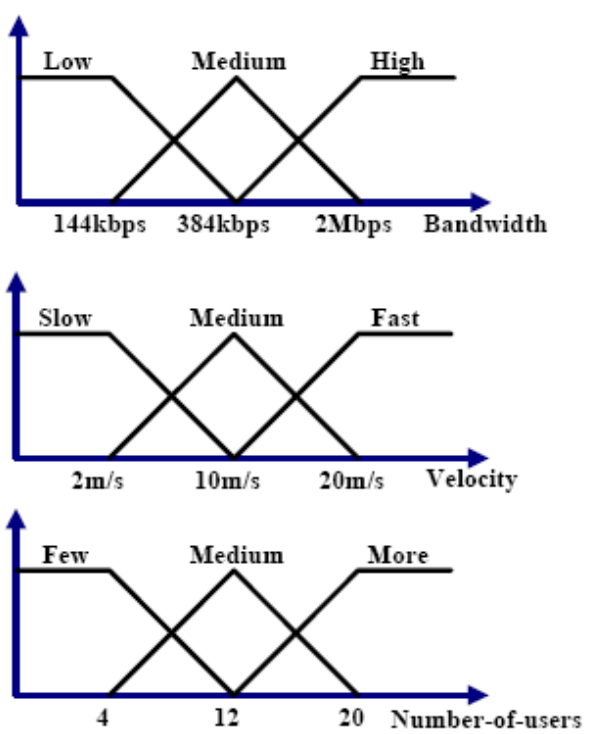

Fig.4. The membership functions of input variables

Increased transmission power means larger transmission range. If the transmission power of a node is too low, then its signal will reach to few neighbors only and its links with those neighbors may have very weak and easy to break. High transmission power of a node will lead to high average number of its neighbors and hence increase the lifetime of its routes. Consequently the following rules are proposed:

R1: If TransPower is high then route must be high

R2: If TransPower is medium then route must be medium

R3: If TransPower is low then route must be low

\subsection{Route Calculation}

The power-aware routing protocols should consider energy consumption from the viewpoints of both the network and the node levels. From the network point of view, the best route is one that minimizes the total transmission power. On the other hand, from the viewpoint of a node, it is one that avoids the nodes with lower power. It is difficult to achieve these two objectives simultaneously. In fact, choosing routes with minimum overall energy consumption, there is a possibility that the chosen paths are routed via a specific node which will in turn result in rapid depletion of energy resource of that node. Hence, the residual battery capacity of each node is a more accurate metric to describe the lifetime of each node. However, we note that the selection of a route that maximizes the minimum residual battery capacities of nodes does not guarantee that the total energy consumption of the selected route is minimized. Hence the selected route may consume more power to transmit user traffic from a source to a destination, which will in turn reduce the lifetime of nodes. ING protocol tries to resolve the problem with regards to these two aspects, i.e. finding a minimum energy route that can maximize the network lifetime.

The transmission power control approach requires power information such as link costs and node costs. In practice, the following issues need to be addressed: (1) How to obtain accurate power information, (2) how much overhead is associated with the energy aware routing, and (3) how to maintain the minimum energy routes in the presence of mobility. 


\subsubsection{TRANSMISSION POWER AND ROUTES}

The routes lifetime used by nodes of ad-hoc network is highly sensitive to the transmission power of those nodes. Transmission power (TransPower) is the strength with which the signal is transmitted. In our system, signal power degradation is modeled by the free space propagation model [8] which states that the received signal strength is:

$$
P_{r}(d)=\frac{P_{t} G_{t} G_{\gamma} \lambda^{2}}{(4 \pi)^{2} d^{2} L}
$$

where Pr and Pt are the receive and transmit powers (in Watts), Gt and Gr are the transmit and receive antenna gains, $\mathrm{d}$ is the transmitter-receiver separation distance, $\mathrm{L}$ is a system loss factor ( $\mathrm{L}=1$ in our simulations which indicates no loss in the system hardware), and lamda is the carrier wavelength (in meters) which related to the carrier frequency by:

$$
\lambda=\frac{c}{f_{c}}
$$

Where $\mathrm{fc}$ is the carrier frequency (in Hertz) and $\mathrm{c}$ is the speed of light $(3 \times 108 \mathrm{~m} / \mathrm{s})$. Assuming a unity gain antenna with a $900 \mathrm{MHz}$ carrier frequency.

\subsubsection{ROUTE DISCOVERY}

Our routing algorithm implements two methods for route discovery and calculation. The home administrative manager can decide to execute one of them, depending on the energy policy that user prefers:

- One of the first proposals in energy related routing metrics is to minimize the per packet consumed energy. The rationale of the metric, called Minimal Total Power Routing metric MTPR in [8], is that this way the overall energy consumption is minimized. Singh et al. [10] formalize this idea as follows: let $e_{i, j}$ denote the energy consumed for transferring a packet from node $i$ to the neighboring node $j$. Then, if the packet has to traverse the path $p$, including nodes $\mathrm{n}_{1}, \ldots \mathrm{n}_{\mathrm{k}}$, the total energy $\mathrm{E}$ required for the packet transfer is:

$$
E=\sum_{i=1}^{k-1} e_{n_{l}, n_{l+1}}
$$

ING addresses this issue and implements the transmission power control mechanism with eight selectable options as follows:

- Option A modifies the header of a route-request packet to include the power used by the sender to transmit the packet. The receiving node uses this information as well as radio power level used to receive the packet to calculate the minimum power required for the successful transmission from the sender to itself. This per hop power information is appended at each intermediate node toward the destination and the destination node informs the source node via the route-reply packet. Then, the source node siply inserts this per hop power information in the data packet header so that all the intermediate nodes as well as the source itself transmit the data packet at the controlled power level. 
- Option F applies the same power control mechanism on the MAC layer's ACK packets.

- Options B, C and D are related to route-cache maintained in the routing algorithm. In Option B, if the source has multiple route candidates in its cache, it calculates the total transmission energy for each possible route based on the power level information obtained via applying Option A and chooses the minimum energy route.

- In Option G, low energy routes are dynamically adjusted when the required transmission power changes due to node mobility.

- Options $\mathrm{E}$ and $\mathrm{H}$ allow non-participating nodes to snoop on packet exchange and to suggest the sender a more energy efficient route at the routing and the MAC layer, respectively.

- The second way is described here after: Chang and Tassiulas in [11] propose a link metric that takes into account the remaining battery capacity and the necessary transmission energy for their Maxi-mum Residual Energy Path (MREP) algorithm. Let $\mathrm{e}_{\mathrm{i}, \mathrm{j}}$ be the energy consumed to send one packet over the link from node $i$ to node $j$, the initial battery energy (capacity), and $\mathrm{E} j$ the residual energy at node $j$. Chang and Tassiulas define two metrics for the link $i->j$ : The remaining energy of a node $\mathrm{d}_{\mathrm{i}, \mathrm{j}}$, defined as

$$
d_{i, j}=\frac{1}{\underline{E}_{j}-e_{i, j}},
$$

and the inverse of the residual capacity of a node in terms of packets that can be delivered with the remaining energy:

$$
d_{i, j}=\frac{e_{i, j}}{\underline{E}_{j}}
$$

Performance evaluation with simulations in the last scenario with highly mobility, both metrics came quite close to a theoretically predicted average node lifetime (theoretical values are calculated using linear programming). Refining their work in [11], they propose a more general formula:

$$
d_{i, j}=e_{i, j}^{x_{1}} \underline{E}_{i}^{-x_{2}} E_{i}^{x_{3}}
$$

where, $x 1, x 2$ and $x 3$ are nonnegative weighting parameters. Simulation evaluation reveals that with reasonable setting of the parameters, the theoretical maximal life-time, the worst-case lifetime, and the transfer reliability can be well approximated.

\section{Performance results}

\subsection{SIMULATION FOR RADIUS OPTIMISATION}

Using Fuzzy Logic Toolbox in Matlab simulator [13], it shows when adopting the same topology as for the next-section testbed that $\mathrm{m}=3$ can guarantee good performances while the proactive region covers most of the network (Figure 5 ) and minimize the overall bit error rate in the network (Figure 6). 
International Journal of Computer Networks \& Communications (IJCNC) Vol.3, No.4, July 2011

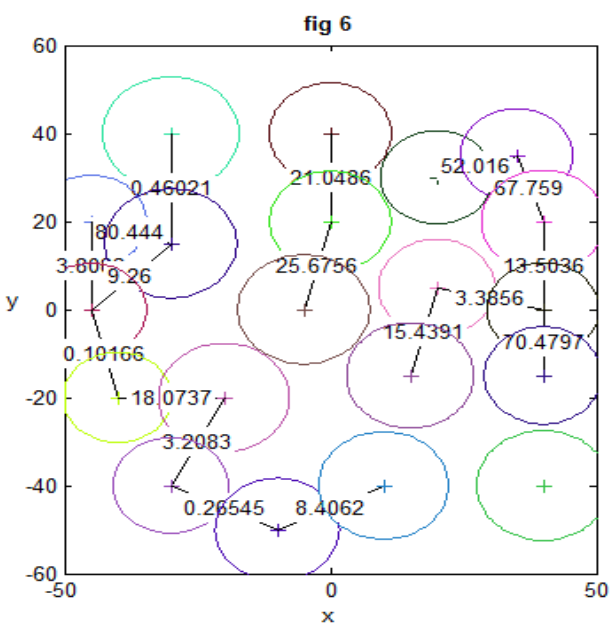

(a) APs radius using fuzzy logic

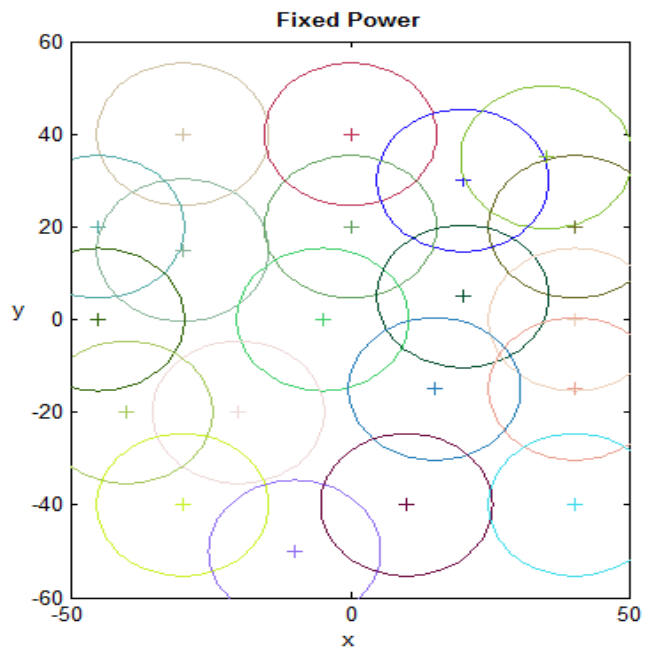

(b) Fixe APs Radius

Figure 5: Matlab Simulations for proactive radius optimisation

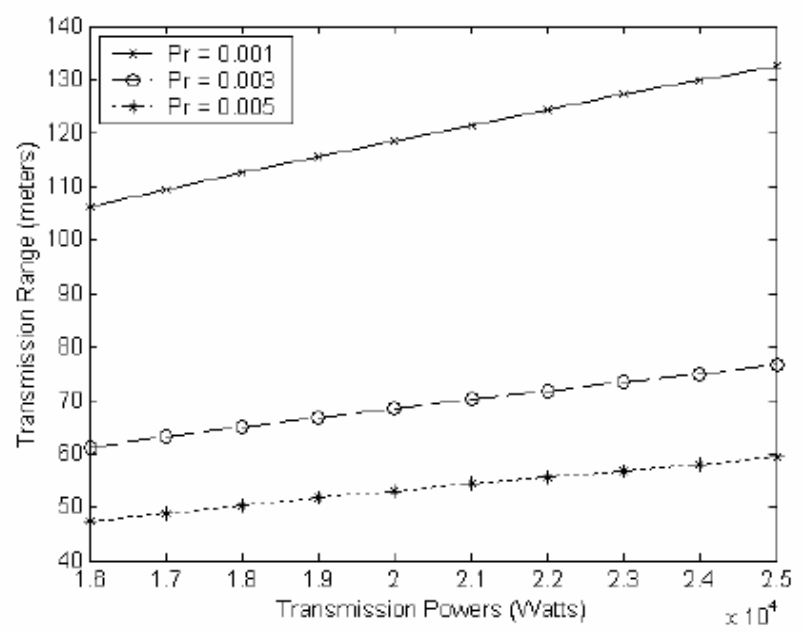

Figure 6: Transmission range under different power constraints

\subsection{PROTOTYPE FOR ING PROTOCOL}

Our prototype implementation of $I N G$ node was implemented as a Linux-2.6.24 kernel module for the Readhat embedded Linux distribution (figure 7). It extends classical AODV protocol for supporting Mesh networks and introduces a new QoS metric for energy aware EA. It implements ING routing between computers equipped with Ethernet or 802.11 devices. The first type of energy saving algorithm was used. 


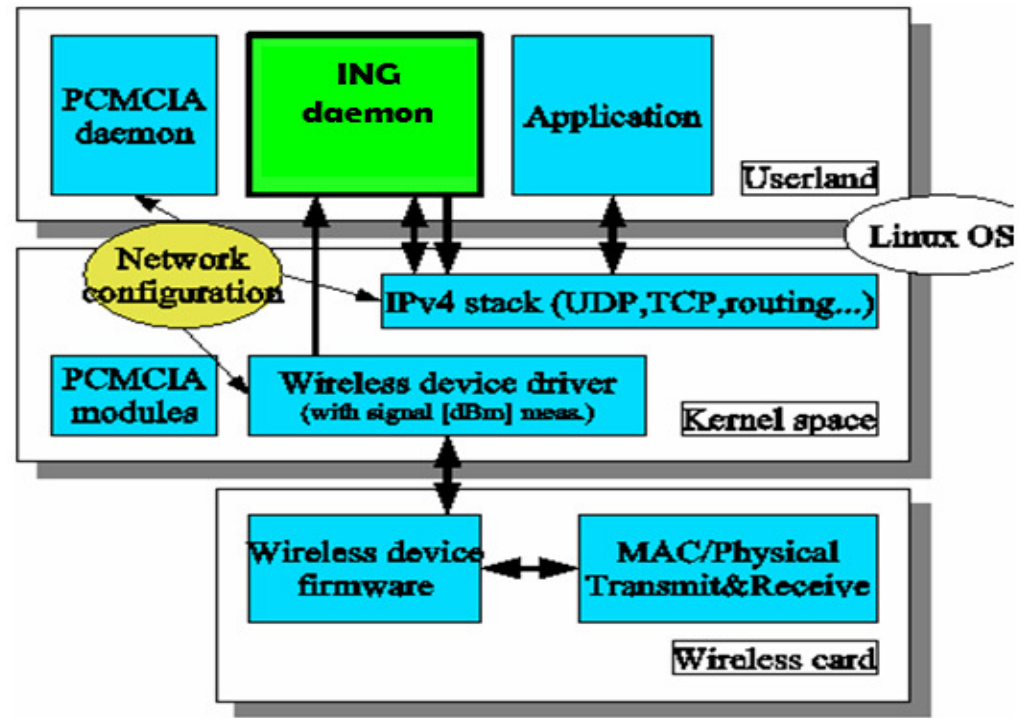

Figure 7: ING implementation in the kernel

The current implementation is a modified version of the AODV implementation [14].It includes all the basic AODV functionality, including Route Discovery (Route Request and Route Reply messages) and Route Maintenance (Route Error messages).We make this choice because of several reasons: it is a open-source project that supports Mac OS X, Windows 98, 2000, XP, Linux, FreeBSD, OpenBSD and NetBSD. It is available for access points that run Linux like the Linksys WRT54G, Asus W1500g, AccessCube or Pocket PCs running Familiar Linux, and ships standard on Metrix kits running Metrix Pebble. It can handle multiple interfaces and is extensible with plug-ins. It supports IPv6 and it is actively developed and used by community networks all over the world.

We have modified AODV in several ways to support routing according to energy-quality metric. These include modifications to Route Discovery and Route Maintenance plus new mechanisms for Metric Maintenance. In brief, the AODV messages are extended to include a 32-bit link-quality metric value for each hop in Source Routes, Route Requests, Route Replies, etc. We do not include a longer description due to space limitations. Our design does not assume that the link-quality metric is symmetric.

The experimental data reported in this paper are the results of measurements we have taken on a 15-node wireless testbed. Our testbed is located on one floor of a fairly typical home building, with the nodes placed in different rooms. The nodes are all Dell or toshiba PCs. Each of these machines has a $2.66 \mathrm{GHz}$ Intel Pentium 4 processor with $512 \mathrm{MB}$ of memory. They all run Microsoft Linux. The TCP stack included with Linux supports the SACK option by default, and we left it enabled. All of our experiments were conducted over IPv4 using statically assigned addresses.

Ranges of nodes vary between 10 and $100 \mathrm{~m}$. The average duration of the connection is about $1 \mathrm{~min}$. Testbed time is $20 \mathrm{~min}$. Some nodes are mobile (approximately 20\%). They selected a random destination within the working area and moved linearly to that location at a speed of $2 \mathrm{~m} / \mathrm{s}$ (walking speed). After reaching their destination, the half of them selected a new random location with no pause. 
It's difficult to precisely define metrics to study the impact of energy awareness on network lifetime. However, we retain two metrics that approximately capture notions of network lifetime: number of dead nodes and packet drop rate. We define the network lifetime as the time taken for $\mathrm{K} \%$ of the home nodes to die due to battery exhaustion. ING is compared to the classical shortest path algorithm "Min-hop".

Energy model has three states where energy is consumed: transmitting, receiving and idle state. Every node starts with initial value which is the level of energy defined by the battery at the beginning of the Testbed. It also has transmitting power (TXpower), receiving power(RXpower) and idle power parameters required by the node's physical layer. Initial energy level is decremented for transmission and reception of packets by TXpower and RXpower. When energy level in a node becomes zero, the node shutdown and is considered as a dead node.

As shown in Figure 8, ING protocol can deliver 20\% to 30\% more packets than classical shortest path algorithm because it takes into consideration the nodes' residual energy when routing packets. It extends by consequence the robustness of the network against nodes failures (confirmed throw Figure 9). We can clearly see that it improves the performance of the ING protocol by giving more precision to the estimation of nodes energy depletion by decreasing packets drop.

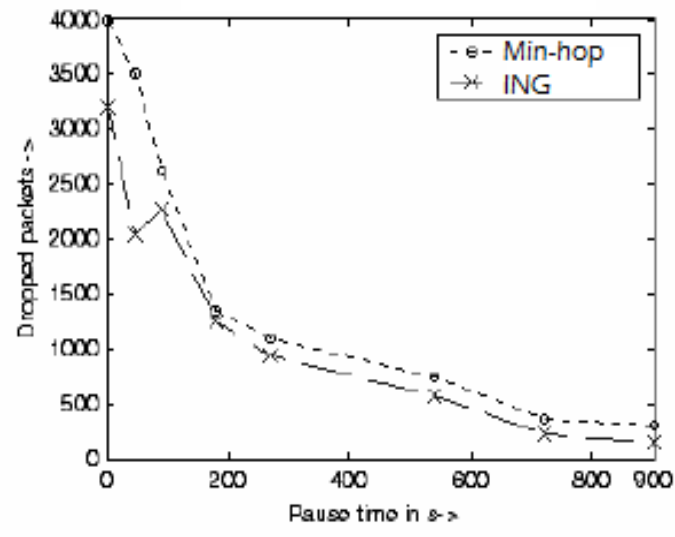

Figure 8: Packet drop rate

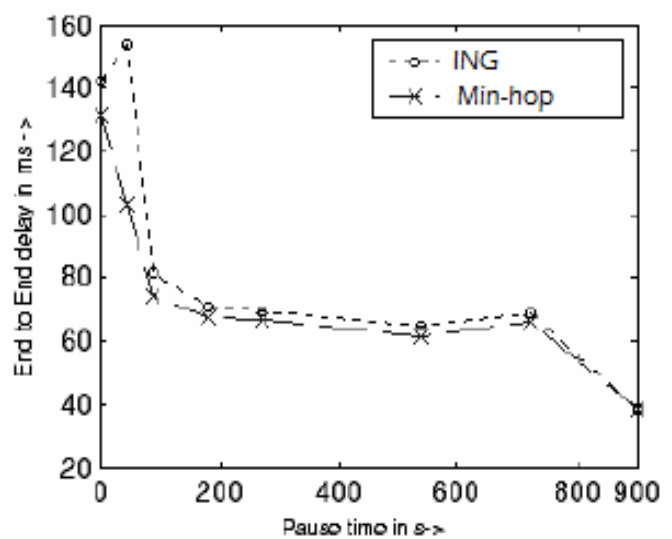

Figure 9: End to end delay

\section{Conclusion}

Although establishing correct and efficient routes is an important design issue in new home networks, a more challenging goal is to provide energy efficient routes because wireless devices operation time is the most critical limiting factor. In this paper, we proposed an energy saving routing protocol adapted for Green home network. ING schema is fully distributed protocol that doesn't need any pre-knowledge of the network. We have shown through simulations and testbed the efficiency of our proposal. Future work will focus on adding security to the home routing algorithm through distributed ciphering.

\section{REFERENCES}

[1] S. Waharte, R. Boutaba, Y.Iraqi and B. Ishibashi, Routing Protocols in Wireless Mesh Networks: Challenges and Design Considerations. Journal of Multimedia Tools Applications, 2008, pp. 285- 303

[2] Draves R, Padhye J, Zill B , "Comparison of routing metrics for static multi-hop wireless networks". Conference on Applications, Technologies, Architectures, and Protocols for Computer Communications 2006 
International Journal of Computer Networks \& Communications (IJCNC) Vol.3, No.4, July 2011

[3] R. Goyal, Divyanshu and Manoj Mishra, "Quality of Service Provisioning in MANET Using a Cross-Layer Approach for Routing"; International journal of computer networks and communication (IJCNC), Vol 3, N.3, 2011

[4] Jiang Q, Manivannan D Routing protocols for sensor networks. Consumer Communications and Networking Conference, (2010, January)

[5] D. B. Johnson, D. A. Maltz, Y. Hu, et J. G. Jetcheva. The Dynamic Source Routing Protocol for Mobile Ad Hoc Networks (DSR). Feb 2002. IETF Internet Draft.

[6] J.J Buckley, E. Eslami, "An introduction to fuzzy logic and fuzzy sets", Book, PhysicaVerlag, Wurzburg, 2002.

[7] Y.F.Wong, W.C.Wong, "A fuzzy decision based routing protocol for mobile ad hoc networks", 10th International Conference on Network 2002, pp 317-322

[8] T. S. Rappaport, “Wireless Communications: Principles \& Practice,” Ch.3, pp. 70- 74, Prentice Hall, 1996

[9] K. Scott and N. Bambos. Routing and channel assignment for low power transmission in PCS. In ICUPC '96, vol. 2, pp. 498-502, 1996

[10] S. Singh, M. Woo, and C. Raghavendra. Power-aware routing in mobile ad hoc net-works. In The Fourth Annual ACM/IEEE International Conference on Mobile Com-puting and Networking, pp. 181-190, 1998.

[11] J. Chang and L. Tassiulas. Maximum lifetime routing in wireless sensor networks. In Proceedings of 37th Annual Allerton Conference on Communication, Control, and Computing, Monticello, IL, September 1999

[12]39. J. Chang and L. Tassiulas. Energy conserving routing in wireless ad-hoc networks. Proceedings of INFOCOM, Israel, March 2000.

[13] Matalb, www.mathworks.fr

[14] AODV Linux, www.antd.nist.gov/wctg/aodv_kernel/ 